\title{
Common Variation in the CYP17A1 and IFIT1 Genes on Chromosome 10 Does Not Contribute to the Risk of Endometriosis
}

\author{
Zhen Zhen Zhao ${ }^{*}$, , Dale R. Nyholt ${ }^{2}$, Lien Le ${ }^{1}$, Susan A. Treloar ${ }^{1}$ and Grant W. Montgomery ${ }^{1}$ \\ ${ }^{I}$ Molecular Epidemiology and ${ }^{2}$ Genetic Epidemiology Laboratories, Queensland Institute of Medical Research, Bris- \\ bane, Queensland, Australia
}

\begin{abstract}
Endometriosis is a complex disease involving multiple susceptibility genes and environmental factors. Our previous studies on endometriosis identified a region of significant linkage on chromosome 10q. Two biological candidate genes (CYP17A1 and IFIT1) located on chromosome 10q, have previously been implicated in endometriosis and/or uterine function. We hypothesized that variation in CYP17A1 and/or IFIT1 could contribute to the risk of endometriosis and may account for some of the linkage signal on chromosome 10q. We genotyped 17 single nucleotide polymorphisms (SNPs) in the CYP17A1 and IFIT1 genes including SNP rs743572 previously associated with endometriosis in 768 endometriosis cases and 768 unrelated controls. We found no evidence for association between endometriosis and individual SNPs or SNP haplotypes in CYP17A1 and IFIT1. Common variation in these genes does not appear to be a major contributor to endometriosis susceptibility in our Australian sample.
\end{abstract}

\section{INTRODUCTION}

Endometriosis is a complex disease involving multiple susceptibility genes and environmental factors [1-4]. Estimates of the population prevalence indicate that endometriosis affects $8-10 \%$ of women of reproductive age $[2,5]$, but the reasons for establishment and progression of endometriosis remain uncertain.

Our previous studies on endometriosis identified a region of significant linkage on chromosome 10q26 [6]. The peak linkage signal is located at $148.75 \mathrm{cM}$ between markers D10S587 and D10S1656 and the 95\% confidence interval (CI) spans a region of 8.5 megabase pairs (Mbp). Gene mapping of plausible candidates may help to define the mechanisms contributing to the genetic susceptibility of endometriosis. Two candidate genes on chromosome 10q, which have previously been implicated in endometriosis and uterine function, are cytochrome P450, family 17, subfamily A, polypeptide 1 (CYP17A1, MIM \#609300) and interferoninduced protein with tetratricopeptide repeats 1 (IFITI, MIM \#147690) [7-9].

Human endometrium is highly responsive to hormonal stimuli during the menstrual cycle with estrogen and progesterone influencing maturational and functional changes in the endometrium. The CYP17A1 gene lies at $104.5 \mathrm{Mbp}$, on the shoulder of our linkage peak on chromosome 10 and encodes the cytochrome P450c17 $\alpha$ enzyme that is involved in estrogen biosynthesis and metabolism [10]. The gene is expressed in human follicles, corpora lutea and endometrial carcinoma cells [11-13]. A number of studies on CYP17A1 variation suggest it could be a genetic biomarker for hormone related diseases $[7,14,15]$. One single nucleotide polymorphism (SNP, rs743572) at the -34 bp position relative to the start codon in the 5'UTR promoter region of

*Address correspondence to this author at the Queensland Institute of Medical, Research, Brisbane, Queensland 4029, Australia;

E-mail: Zhen.Zhao@qimr.edu.au
CYP17A1 has been commonly studied. The SNP was thought to be associated with a Sp-1 binding site that would lead to higher gene expression in male baldness [16]. In patients with endometrial cancer, a marked decrease in the A2 allele of CYP17A1 was seen when compared with normal controls [7]. A subsequent study on 119 endometriosis cases and 108 normal controls demonstrated that the CYP17AI allele was associated with an increased risk of endometriosis in a Chinese population [8], but lack of association between endometriosis and the CYP17A1 polymorphism was observed in UK and Japanese populations [17].

The IFIT1 gene is located at $91.1 \mathrm{Mbp}$, close to the CYP17A1 locus just outside the $95 \%$ confidence region for our linkage peak. However the linkage peak is broad and there is evidence for linkage and association with endometriosis in Puerto Rican families at marker D10S677 [18], which is located at $113.34 \mathrm{cM}(95.95 \mathrm{Mb})$ close to the IFIT1 locus. During early pregnancy, IFITl is highly expressed upon stimulation with interferon tau (IFNT), a type I interferon produced by the conceptus trophectoderm $[9,19]$. As an endometrial gene, IFIT1 is believed to respond to hormonal stimulation and may have a functional role for uterine support of peri-implantation conceptus survival, growth, and implantation [9]. Although IFIT1 has been studied in the sheep model, it is unknown if variants in IFITI contribute to risk of human endometriosis.

We previously excluded association between endometriosis and candidate genes under the linkage peak including empty spiracles homeobox 2 (EMX2), phosphatase and tensin homolog $(P T E N)$ and fibroblast growth factor receptor 2 (FGFR2) in our Australian sample [20,21]. To continue to search for the gene or genes contributing to the linkage peak, we examined variation in IFIT1 and/or CYP17Al to determine whether those genes contribute to the risk of endometriosis and may account for some of the linkage signal on chromosome 10q. 


\section{MATERIALS AND METHODS}

\section{Participants and Sample Collection}

The project was approved by the Human Research Ethics Committee of the Queensland Institute of Medical Research and the Australian Twin Registry. Women with surgically confirmed endometriosis were selected from each of 768 Australian affected sister pair families as previously described [20]. The sister with the most severe stage of disease was chosen for genotyping. Disease severity was assessed retrospectively from medical records using the revised American Fertility Society (rAFS) classification system [22]. Sixty one percent of cases were classified with minimal to mild endometriosis (rAFS stages I/II). The remaining 39\% of cases with moderate to severe (rAFS stages III/IV) endometriosis were more likely to have ovarian endometriosis. A total of 645 cases $(84 \%)$ were diagnosed at laparoscopy; the remaining cases were mostly diagnosed at hysterectomy, or in a small number of cases at laparotomy or during another procedure.

The controls were 768 unrelated women who had volunteered for a twin study of gynaecological health [2]. Controls were selected after consideration of the competing issues of ascertainment bias from clinic controls and presence of undiagnosed cases. They were selected from women who selfreported they had never been diagnosed with endometriosis and were therefore considered to be at low risk of having endometriosis. Twins had been asked simply 'have you had endometriosis?'[2]. Additional information from medical records was used where available. Women were also asked whether they had ever had a laparoscopy and/or a hysterectomy and the reasons for each. About $14 \%$ of control women reported having a hysterectomy and/or laparoscopy. No evidence of endometriosis was reported at any of these procedures in our control sample [20]. The mean ages ( \pm SD) of the cases and controls at the time of data collection were $35.6 \pm 9.1$ years $($ range $=17-65)$ and $45.7 \pm 12.2($ range $=$ 29-90) years respectively. Genomic DNAs were extracted [23], and diluted to a working concentration of $2.5 \mathrm{ng} / \mu \mathrm{l}$. The case and control DNAs were randomly placed in 384well PCR plates.

\section{SNP Selection}

We selected 7 SNPs in the CYP17A1 gene based upon the allelic association results in endometriosis [8] and allele frequency information from the National Center for Biotechnology Information (NCBI, http://www.ncbi.nlm.nih.gov/). Ten SNPs were selected in the IFIT1 gene on the basis of the allelic frequency information and SNP distribution across the gene. The chosen CYP17Al SNP list comprised two promoter, two intronic, two coding exonic and one 3'UTR SNPs. One 5'UTR promoter, seven intronic and two coding exonic SNPs were chosen in the IFIT1 gene. All SNP sequences were downloaded from the Chip Bioinformatics database (http://snpper.chip.org/) and the sequences were cross checked in NCBI and Sequenom RealSNP databases (https://www.realsnp.com/) before assay design.

\section{Genotyping}

Multiplex assays were designed using the Sequenom MassARRAY Assay Design software (version 3.0). SNPs were typed using Sequenom iPLEX $^{\mathrm{TM}}$ chemistry on a MALDI-TOF Mass Spectrometer. The $2.5 \mu \mathrm{L}$ PCR reactions were performed in standard 384-well plates using $10 \mathrm{ng}$ genomic DNA, 0.5 unit of Taq polymerase (Qiagen, Valencia, CA), $500 \mu \mathrm{mol}$ of each dNTP, and $100 \mathrm{nmol}$ of each PCR primers. Standard PCR thermal cycling conditions and postPCR extension reactions were carried out as described previously [24]. The iPLEX reaction products were desalted by diluting samples with $15 \mu 1$ of water and adding $3 \mu 1$ of resin. The products were spotted on a SpectroChip (Sequenom), and data were processed and analysed in a Compact Mass Spectrometer by MassARRAY Workstation (version 3.4) software (Sequenom).

\section{Statistical Analysis}

The genotypes were inspected and results were tested for departures from Hardy-Weinberg equilibrium (HWE) separately for cases and controls using Haploview version 3.32 (Whitehead Institute for Biomedical Research, USA). The PLINK program (http://pngu.mgh.harvard.edu/purcell/ plink/) was used to test association between endometriosis and individual SNPs. Global p-values were obtained for each marker or each haplotype by performing 10,000 permutation tests. Haplotype frequencies, linkage disequilibrium (LD) estimates and analysis were determined by Haploview [25] using the default method of Gabriel [26]. A global p-value $<0.05$ was considered to be statistically significant.

We performed power calculations for our case-control study assuming a disease (endometriosis) prevalence of 10\% using the Genetic Power Calculator [27]. Power calculations were based on 768 unrelated cases and 768 unrelated controls using a significance threshold $(\alpha)$ of $P=0.01$.

\section{RESULTS}

Seven SNPs in the CYP17Al gene and ten SNPs in the IFIT1 gene were typed in 768 endometriosis cases and 768 unrelated controls. All SNPs were in Hardy-Weinberg equilibrium. The minor allele frequencies of the CYP17A1 SNPs ranged from 0.230 to 0.414 in cases and from 0.200 to 0.443 in controls. The minor allele frequencies of the IFIT1 SNPs ranged from 0.058 to 0.484 in cases and from 0.072 to 0.498 in controls (Table 1). The minor allele frequency for the key SNP (rs743572) in the CYP17Al gene was 0.386 and 0.397 in cases and controls, respectively. There was no significant difference in allele frequency between cases and controls for this key SNP (Table 1). SNP rs2486758, at the -362 bp position relative to the start codon in the 5'UTR promoter region of CYP17Al gene showed nominal evidence of association $(P<0.05)$. However, the difference in allele frequency between cases and controls was small and the effects were not significant after correcting for multiple testing of all SNPs.

The positions of the SNPs genotyped in the IFITI gene and the CYP17Al gene are shown in Fig. (1a). A linkage disequilibrium plot of SNPs and common haplotype blocks for the both genes are also shown in Fig. (1b,c). We found no evidence for association between endometriosis and individual SNPs in either IFITI or CYPI7A1 for either the allelic or the genotypic association tests (Table 1).

Stratification of cases according to stage of disease (469 Stage A cases and 768 controls) gave a best point-wise $P$ value of 0.02 for SNP rs619824 in the CYP17Al gene, but the global result correcting for multiple tests was nonsignificant $(P=0.58)$. Analysis of 296 cases diagnosed with 
Table 1. Association Analysis of 17 SNPs Across the IFIT1 and CYP17A1 Gene Locus Genotyped in 768 Endometriosis Cases and 768 Controls

\begin{tabular}{|c|c|c|c|c|c|c|c|c|c|}
\hline dbSNP ID & SNP Position & Gene(s) & Role & Alleles & MAF-Cases & MAF-Controls & $\chi^{2}$ & $P$ Value & OR \\
\hline rs304478 & chr10:91140902 & IFIT1 & Promoter & $\mathrm{G}>\mathrm{T}$ & 0.456 & 0.463 & 0.139 & 0.709 & 0.972 \\
\hline rs303218 & chr10:91142573 & IFIT1 & Intron & $\mathrm{G}>\mathrm{A}$ & 0.175 & 0.186 & 0.620 & 0.431 & 0.929 \\
\hline rs303217 & chr10:91143679 & IFIT1 & Intron & $\mathrm{T}>\mathrm{C}$ & 0.470 & 0.469 & 0.002 & 0.968 & 1.003 \\
\hline rs303216 & chr10:91145166 & IFIT1 & Intron & $\mathrm{C}>\mathrm{T}$ & 0.169 & 0.182 & 0.858 & 0.354 & 0.915 \\
\hline rs303215 & chr10:91146811 & IFIT1 & Intron & $\mathrm{T}>\mathrm{C}$ & 0.170 & 0.182 & 0.754 & 0.385 & 0.921 \\
\hline rs304484 & chr10:91149303 & IFIT1 & Intron & $\mathrm{G}>\mathrm{T}$ & 0.231 & 0.244 & 0.770 & 0.380 & 0.928 \\
\hline rs304485 & chr10:91149890 & IFIT1 & Intron & $\mathrm{T}>\mathrm{A}$ & 0.484 & 0.498 & 0.578 & 0.447 & 0.946 \\
\hline rs303212 & chr10:91151335 & IFIT1 & Intron & $\mathrm{T}>\mathrm{C}$ & 0.232 & 0.241 & 0.357 & 0.550 & 0.950 \\
\hline rs303211 & chr10:91152477 & IFIT1 & Coding exon & $\mathrm{G}>\mathrm{A}$ & 0.058 & 0.072 & 2.581 & 0.108 & 0.790 \\
\hline rs 303210 & chr10:91152657 & IFIT1 & Coding exon & $\mathrm{C}>\mathrm{T}$ & 0.169 & 0.182 & 0.822 & 0.365 & 0.916 \\
\hline rs619824 & chr10:104571278 & CYP17A1 & 3' UTR & $\mathrm{G}>\mathrm{T}$ & 0.414 & 0.443 & 2.633 & 0.105 & 0.888 \\
\hline rs3740397 & chr10:104582665 & CYP17A1 & Intron (boundary) & $\mathrm{C}>\mathrm{G}$ & 0.375 & 0.396 & 1.404 & 0.236 & 0.916 \\
\hline rs4919687 & chr10:104585238 & CYP17A1 & Intron (boundary) & $\mathrm{G}>\mathrm{A}$ & 0.347 & 0.359 & 0.539 & 0.463 & 0.946 \\
\hline rs6163 & chr10:104586914 & CYP17A1 & Coding exon & $\mathrm{C}>\mathrm{A}$ & 0.376 & 0.395 & 1.058 & 0.304 & 0.926 \\
\hline rs6162 & chr10:104586971 & CYP17A1 & Coding exon & $\mathrm{G}>\mathrm{A}$ & 0.389 & 0.403 & 0.573 & 0.449 & 0.945 \\
\hline rs743572 & chr10:104587142 & CYP17A1 & 5' UTR & $\mathrm{T}>\mathrm{C}$ & 0.386 & 0.397 & 0.376 & 0.540 & 0.954 \\
\hline rs 2486758 & chr10:104587470 & CYP17A1 & 5' UTR & $\mathrm{T}>\mathrm{C}$ & 0.230 & 0.200 & 3.870 & 0.049 & 1.189 \\
\hline
\end{tabular}

MAF: minor allele frequency.

dbSNP ID: database SNP identification.

UTR: untranslated region.

stage B and 768 controls, showed no significant differences between cases and controls for any SNPs typed in the study.

\section{DISCUSSION}

Our results do not support an association between endometriosis and common variation in either CYP17Al or IFIT1. Although both genes are biological candidates for endometriosis and are located near the peak of our linkage signal on chromosome 10, there was no evidence that variants in either gene were associated with endometriosis or contribute to the linkage signal on chromosome 10q.

Genetic studies of CYP17A1 variants to date have followed a defined biological hypothesis suggesting the 5'UTR promoter region SNP (rs743572) is associated with gene expression [16]. While the $\mathrm{T}$ allele (rs743572) was found to be associated with increased risk of endometriosis in a study of Chinese women [14], there was no association between CYP17Al variants and endometriosis in studies in Brazilian, UK or Japanese populations [17, 28, 29]. Endometriosis is a sex steroid-dependent disease [30]. CYP17A1 is involved in estrogen biosynthesis and metabolism so that certain genetic polymorphisms in the gene could be associated with increased risk of developing endometriosis. The differences in the results may relate to study power (sample size) or population differences. We estimated power for our case-control study based on total sample of 768 cases and 768 controls. There is over $80 \%$ power to detect allele frequency of 0.05 , 0.25 and 0.5 contributing a dominant genotype relative risk (GRR) of 1.7, 1.5 and 1.8, respectively. In contrast, when the sample size changed to total sample of 100 cases and 100 controls, there is only $10 \%$ power to detect allele frequency of $0.05,0.25$ and 0.5 contributing the similar genotype relative risk as stated above. These calculations demonstrate our sample has high power to detect novel gene associations of moderate effect. However, because our cases are highly selected in terms of family history, compared to a standard case-control association study, our sample will have considerably more power to detect gene associations.

We examined variation in CYP17Al in cases from families contributing to the linkage peak in this region of chromosome 10q and genotyped 7 CYP17Al SNPs including rs743572 in 768 endometriosis and 768 controls. We found no evidence for association with either SNP rs743572 in the promoter of CYP17A1 or the other 6 SNPs in the gene in our Australian sample. Strong linkage disequilibrium (LD) was detected between SNP rs743572 and the other three SNPs in the gene (rs3740397, $r^{2}=0.948 ;$ rs6163, $r^{2}=0.958 ;$ rs6162, $r^{2}=0.919$ ) in our sample. It is unlikely that any asymptomatic cases present in the control samples would affect the conclusion from this study for a disease with a prevalence of $8-10 \%$ [31].

IFIT1 is a hormonally responsive gene expressed in sheep endometrium following stimulation with IFNT [9, 19]. To test for association between IFIT1 variants and human endometriosis we typed 10 common IFIT1 SNPs in our 768 endometriosis cases and 768 unrelated controls. There was no evidence for association between the individual SNPs and endometriosis. Analysis of the SNPs across the IFIT1 locus 


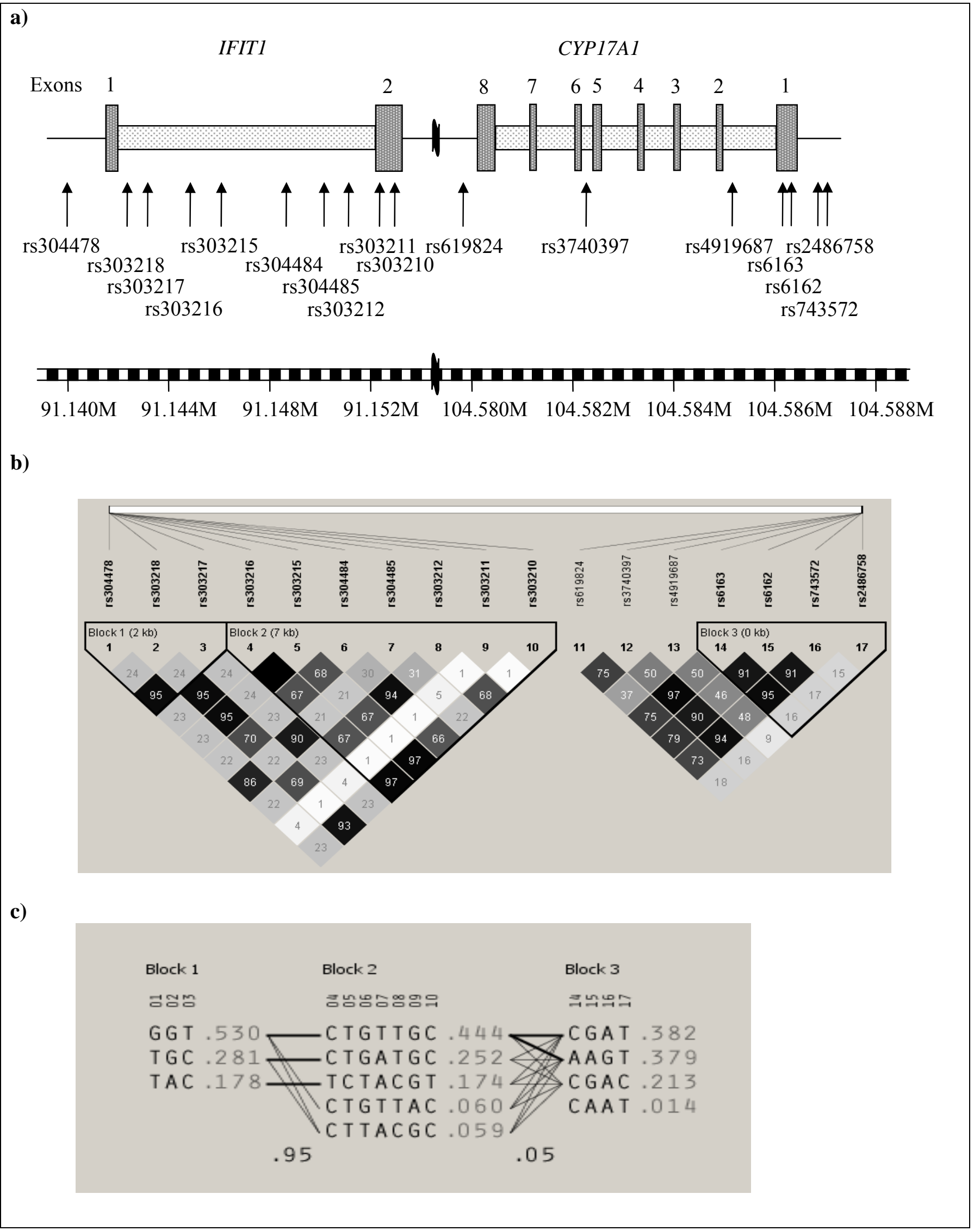

Fig. (1). Variants typed in the human IFIT1 and CYP17A1 genes (a) the genomic structure of the IFIT1 and CYP17A1 genes showing the location of the 17 SNPs genotyped (b) the linkage disequilibrium plot of single nucleotide polymorphism estimated as $\gamma^{2}$ using Haploview (c) common haplotypes and association analysis with endometriosis. Shading key: white $\gamma^{2}=0$; shades of grey $0>\gamma^{2}<1$; black $\gamma^{2}=1$ 
showed strong linkage disequilibrium, with two haplotype blocks and three major haplotypes accounting for $87 \%$ of the chromosomes in our case samples. Our data also show strong linkage disequilibrium (LD) in two blocks covering most of the gene in our samples (Fig. 1b). We did not find any evidence for association between SNP haplotype frequencies and endometriosis. IFIT1 may be important in uterine development and function, but common variation is not associated with risk of endometriosis.

In this study, we examined the association between endometriosis and individual common SNPs and haplotypes in the IFIT1 and CYP17Al genes on chromosome 10q in an Australian population including a functional SNP in the CYP17A1 promoter region. Our data does not provide evidence supporting an association between common variation in the IFIT1 and CYP17AI genes and endometriosis susceptibility. We have previously demonstrated significant linkage to endometriosis on chromosome 10q [6]. Results of the present study demonstrate that variation in the CYP17A1 and IFIT1 genes does not explain linkage to endometriosis in this region of chromosome 10 . Both genes are good candidates for endometriosis but the linkage region spans approximately 8.5 million DNA base pairs and contains over 50 known genes within the $95 \%$ confidence interval for the linkage peak. Many of these other genes can also be considered candidates for endometriosis and variation in one or more of these genes may explain the linkage signal in this region. We conclude that common variants in the IFIT1 and CYP17A1 genes do not play a key role in the pathogenesis of endometriosis.

\section{ACKNOWLEDGMENTS}

We thank Dr. Daniel T. O'Connor for confirmation of diagnosis and staging of disease from clinical records of 295 cases; Barbara Haddon for co-ordination of family recruitment, blood and phenotype collection; Anjali Henders, Megan Campbell and staff of the Molecular Epidemiology Laboratory for sample processing and DNA preparation. This study was supported by the Australian Government's Cooperative Research Centre's Program and National Health and Medical Research Council of Australia (339430, 339446).

\section{REFERENCES}

[1] Kennedy S, Mardon H, Barlow D. Familial endometriosis. J Assist Reprod Genet 1995; 12: 32-4.

[2] Treloar SA, O'Connor DT, O'Connor VM, Martin NG. Genetic influences on endometriosis in an Australian twin sample. Fertil Steril 1999; 71: 701-10.

[3] Hadfield RM, Mardon HJ, Barlow DH, Kennedy SH. Endometriosis in monozygotic twins. Fertil Steril 1997; 68: 941-2.

[4] Stefansson H, Geirsson RT, Steinthorsdottir V, et al. Genetic factors contribute to the risk of developing endometriosis. Hum Reprod 2002; 17: 555-9.

[5] Eskenazi B, Warner ML. Epidemiology of endometriosis. Obstet Gynecol Clin North Am 1997; 24: 235-58.
Treloar SA, Wicks J, Nyholt DR, et al. Genomewide linkage study in 1,176 affected sister pair families identifies a significant susceptibility locus for endometriosis on chromosome 10q26. Am J Hum Genet 2005; 77: 365-76.

[7] Haiman CA, Hankinson SE, Colditz GA, Hunter DJ, De Vivo I. A polymorphism in CYP17 and endometrial cancer risk. Cancer Res 2001; 61: 3955-60.

[8] Hsieh YY, Chang CC, Tsai FJ, Lin CC, Tsai CH. Estrogen receptor alpha dinucleotide repeat and cytochrome $\mathrm{P} 450 \mathrm{c} 17$ alpha gene polymorphisms are associated with susceptibility to endometriosis. Fertil Steril 2005; 83: 567-72.

[9] Gray CA, Abbey CA, Beremand PD, et al. Identification of endometrial genes regulated by early pregnancy, progesterone, and interferon tau in the ovine uterus. Biol Reprod 2006; 74: 383-94.

[10] Picado-Leonard J, Miller WL. Cloning and sequence of the human gene for P450c17 (steroid 17 alpha-hydroxylase/17,20 lyase): similarity with the gene for P450c21. DNA 1987; 6: 439-48.

[11] Doody KJ, Lorence MC, Mason JI, Simpson ER. Expression of messenger ribonucleic acid species encoding steroidogenic enzymes in human follicles and corpora lutea throughout the menstrual cycle. J Clin Endocrinol Metab 1990; 70: 1041-5.

[12] Suzuki T, Sasano H, Tamura M, et al. Temporal and spatial localization of steroidogenic enzymes in premenopausal human ovaries: in situ hybridization and immunohistochemical study. Mol Cell Endocrinol 1993; 97: 135-43.

[13] Sugawara T, Nomura E, Fujimoto S. Expression of enzyme associated with steroid hormone synthesis and local production of steroid hormone in endometrial carcinoma cells. J Endocrinol 2004; 180: 135-44.

[14] Hsieh YY, Chang CC, Tsai FJ, Lin CC, Tsai CH. Cytochrome $\mathrm{P} 450 \mathrm{c} 17$ alpha 5'-untranslated region *T/C polymorphism in endometriosis. J Genet 2004; 83: 189-92.

[15] Olson SH, Bandera EV, Orlow I. Variants in estrogen biosynthesis genes, sex steroid hormone levels, and endometrial cancer: a HuGE review. Am J Epidemiol 2007; 165: 235-45.

[16] Carey AH, Waterworth D, Patel K, et al. Polycystic ovaries and premature male pattern baldness are associated with one allele of the steroid metabolism gene CYP17. Hum Mol Genet 1994; 3: 1873-6.

[17] Asghar T, Yoshida S, Nakago S, et al. Lack of association between endometriosis and the CYP17 MspA1 polymorphism in UK and Japanese populations. Gynecol Endocrinol 2005; 20: 59-63.

[18] Flores I, Mandal D, Bailey-Wilson J. Search for endometriosis susceptibility genes in Puerto Rico. Abstract 1905, American Society of Human Genetics meeting, Toronto. 2004.

[19] Kim S, Choi Y, Bazer FW, Spencer TE. Identification of genes in the ovine endometrium regulated by interferon tau independent of signal transducer and activator of transcription 1. Endocrinology 2003; 144: 5203-14.

[20] Treloar SA, Zhao ZZ, Le L, et al. Variants in EMX2 and PTEN do not contribute to risk of endometriosis. Mol Hum Reprod 2007; 13: 587-94.

[21] Zhao ZZ, Pollock PM, Thomas S, et al. Common variation in the fibroblast growth factor receptor 2 gene is not associated with endometriosis risk. Hum Reprod 2008; 23: 1661-8.

[22] Revised American Fertility Society classification of endometriosis: 1985. Fertil Steril 1985; 43: 351-2.

[23] Miller SA, Dykes DD, Polesky HF. A simple salting out procedure for extracting DNA from human nucleated cells. Nucleic Acids Res 1988; 16: 1215 .

[24] Zhao ZZ, Nyholt DR, Le L, et al. KRAS variation and risk of endometriosis. Mol Hum Reprod 2006; 12: 671-6.

[25] Barrett JC, Fry B, Maller J, Daly MJ Haploview: analysis and visualization of LD and haplotype maps. Bioinformatics 2005; 21 : 263-5.

[26] Gabriel SB, Schaffner SF, Nguyen H, et al. The structure of haplotype blocks in the human genome. Science 2002; 296: 2225-9. 
[27] Purcell S, Cherny SS, Sham PC. Genetic Power Calculator: designof linkage and association genetic mapping studies of complex traits. Bioinformatics 2003; 19: 149-50.

[28] Kado N, Kitawaki J, Obayashi H, et al. Association of the CYP17 gene and CYP19 gene polymorphisms with risk of endometriosis in Japanese women. Hum Reprod 2002; 17: 897-902.

[29] De Carvalho CV, Nogueira-De-Souza NC, Costa AM, et al. Genetic polymorphisms of cytochrome P450cl7alpha (CYP17) and progesterone receptor genes (PROGINS) in the assessment of endometriosis risk. Gynecol Endocrinol 2007; 23: 29-33.

[30] Guo SW. Association of endometriosis risk and genetic polymorphisms involving sex steroid biosynthesis and their receptors: a meta-analysis. Gynecol Obstet Invest 2006; 61: 90-105.

[31] Moskvina V, Holmans P, Schmidt KM, Craddock N. Design of case-controls studies with unscreened controls. Ann Hum Genet 2005; 69: 566-76.

(C) Zhao et al.; Licensee Bentham Open.

This is an open access article distributed under the terms of the Creative Commons Attribution License (http://creativecommons.org/licenses/by/2.5/), which permits unrestrictive use, distribution, and reproduction in any medium, provided the original work is properly cited. 